\title{
Utilisation et Gestion des Pesticides dans les Zones Agricoles Urbaines, Périurbaines et Rurales de la Préfecture de Meknès, Maroc
}

\author{
Ibrahim El Ghazi \\ Université Moulay Ismail, Cluster des Compétences \\ Environnement et santé, Meknès, Maroc \\ Université Catholique de Louvain, Belgique \\ Janvier Egah \\ Université Catholique de Louvain, Belgique \\ Laboratoire Société- Environnement (LaSEn), Faculté d'Agronomie, \\ Université de Parakou, Bénin

\section{Berni Imane, \\ Aziza Menouni, \\ Mohammed Amane} \\ Université Moulay Ismail, Cluster des Compétences \\ Environnement et santé, Meknès, Maroc \\ Marie-Paule Kestemont \\ Université Catholique de Louvain, Belgique \\ Samir El Jaafari \\ Université Moulay Ismail, Cluster des Compétences \\ Environnement et santé, Meknès, Maroc
}

Doi:10.19044/esj.2021.v17n34p94

Submitted: 26 August 2021

Accepted: 15 September 2021

Published: 30 September 2021
Copyright 2021 Author(s)

Under Creative Commons BY-NC-ND 4.0 OPEN ACCESS

Cite As:

El Ghazi I., Egah J., Imane B., Menouni A., Amane M., Kestemont M-P. \& El Jaafari S. (2021). Utilisation et Gestion des Pesticides dans les Zones Agricoles Urbaines, Périurbaines et Rurales de la Préfecture de Meknès, Maroc. European Scientific Journal, ESJ, 17(34), 94. https://doi.org/10.19044/esj.2021.v17n34p94

\section{Résumé}

L'utilisation inappropriée des pesticides a des effets négatifs sur l'Homme, la faune et la flore. Le manque d'une base de données sur l'utilisation des pesticides dans la Préfecture de Meknès (Maroc), nous a conduit à réaliser des enquêtes auprès de 120 agriculteurs choisis de façon aléatoire stratifiée dans cette localité. Le questionnaire a porté sur les 
pesticides utilisés, les moyens de protection des agriculteurs pendant la préparation de la bouillie et l'usage des pesticides, la gestion des emballages des pesticides, le rinçage des pulvérisateurs et l'élimination de la bouillie restante. Les résultats de l'enquête auprès des enquêtés ont montré que les pesticides les plus fréquemment utilisés sont essentiellement des herbicides et des fongicides à des taux respectifs de $44 \%$ et $29 \%$. Les familles chimiques des pesticides les plus recensées durant cette enquête sont celles des triazoles (21\%) et des organophosphorés (17\%). Le glyphosate a été la matière active la plus utilisée (13\%). Les 120 agriculteurs interrogés traitaient sans protection et vivaient à côté des parcelles. Il est essentiel donc d'accentuer la sensibilisation des agriculteurs sur l'utilisation rationnelle des pesticides et l'adoption des techniques alternatives pour diminuer les impacts sur la santé et l'environnement.

Mots clés: Pesticides, Herbicides, Glyphosate, Enquête, Meknès, Maroc

\title{
The Use and Management of Pesticides in Urban, Peri-Urban and Rural Agricultural Areas of The Prefecture of Meknes, Morocco
}

\author{
Ibrahim El Ghazi \\ Université Moulay Ismail, Cluster des Compétences \\ Environnement et santé, Meknès, Maroc \\ Université Catholique de Louvain, Belgique \\ Janvier Egah \\ Université Catholique de Louvain, Belgique \\ Laboratoire Société- Environnement (LaSEn), Faculté d'Agronomie, \\ Université de Parakou, Bénin \\ Berni Imane, \\ Aziza Menouni, \\ Mohammed Amane \\ Université Moulay Ismail, Cluster des Compétences \\ Environnement et santé, Meknès, Maroc \\ Marie-Paule Kestemont \\ Université Catholique de Louvain, Belgique \\ Samir El Jaafari \\ Université Moulay Ismail, Cluster des Compétences \\ Environnement et santé, Meknès, Maroc
}




\begin{abstract}
The inappropriate use of pesticides has negative impact on humans, fauna and flora. The lack of a database on pesticides use in the Prefecture of Meknes (Morocco), led us to conduct surveys in this locality. A stratified of 120 farmers was randomly selected. The questionnaire focused on pesticides used, farmers' protective measures during the slurry preparation and pesticides use, management of pesticide packaging, sprayers rinsing and disposal of leftover slurry. The results of this survey show that the herbicides and fungicides were the main pesticides used at $44 \%$ and $29 \%$ respectively. The chemical families of pesticides most frequently identified during this survey were triazoles (21\%) and organophosphates (17\%). Glyphosate is the most consumed active ingredient (13\%). The 120 farmers interviewed treated without protection and lived next to the plots. It is therefore essential to accentuate farmers' awareness of the rational use of pesticides and the adoption of alternative techniques to reduce the impact on health and environment.
\end{abstract}

Keywords: Pesticides, Herbicides, Glyphosate, Survey, Meknes, Morocco

\title{
Introduction
}

Le Maroc est très dépendant du secteur agricole. En effet, environ 40\% de la population laborieuse travaille dans l'agriculture et $14 \%$ du Produit Intérieur Brut (PIB) est généré par ce secteur (MAPM, 2014). En outre, l'agriculture assure l'approvisionnement alimentaire de la population marocaine et est destinée également pour l'exportation. La culture agricole conventionnelle des céréales, des fruits et des légumes au Maroc comme dans la plupart des autres pays du monde est associée à l'utilisation des pesticides. Ces pesticides sont des substances toxiques qui permettent d'éliminer les plantes, les insectes et les champignons nuisibles. Lorsqu'ils sont utilisés de manière abusive et inappropriée, des résidus de produits toxiques se forment dans les denrées cultivées (Morillon, 2016; Assogba-Komlan et al, 2007 ; Carvalho, 2006 ; FAO, 1978). Aussi la biodiversité en pâtit-elle également (El Bakouri, 2006 ; Pazou et al., 2006) et les sources d'eaux sont susceptibles d'être contaminées (Berni et al., 2021 a ; Naamane et al, 2020 ; Maldani et al., 2017 ; El Azzouzi et al., 2014 ; Detroux, 1996).

La forte demande des produits alimentaires par les consommateurs et la nécessité de maintenir les prix des denrées alimentaires aussi bas que possible exigent une production intense qui pousse à l'utilisation massive des produits phytosanitaires pour booster la production et améliorer le rendement. L’usage exagérée de ces produits est susceptible d'affecter négativement la qualité de l'air (Guiral et al., 2016 ; Chahine, 2011 ; FOCUS, 2008; Houze, 2003), du sol (Raffa et Chiampo 2021 ; Barriuso et al., 2005 ; Bouzoubaa, 
2002 ; Van Jaarsveld et al., 1999), de l'eau (Berni et al., 2021b), la biodiversité (Cusaac et al., 2015 ; Brühl et al., 2011 ; Dinehart et al., 2009) et la santé à la fois des applicateurs de ces produits phytosanitaires et des consommateurs des denrées agricoles traités (Berni et al., 2021 b; Saadane, 2018 ; Belhad et al., 2017 ; Morillon, 2016; Idrissi et al., 2010 ; Carvalho, 2006 ; Wade, 2003).

$\mathrm{Au}$ Maroc, selon les statistiques de l'Office des Changes, les importations des produits phytosanitaires sont en constante augmentation depuis l'année 2005 (El Ouilani, 2011). Elles avoisinent 12000 tonnes par an et représentent un chiffre d'affaire estimé à 90 millions de Dollars US par an (Mojtahid, 2006). Par ailleurs, selon le Centre Antipoison, les pesticides représentent la deuxième cause d'intoxication au niveau national, tout en sachant que près de 20000 décès accidentels (WHO, 1990) et 385 millions de cas d'intoxication aiguë non intentionnelle sont liés à l'exposition aux pesticides chaque année au niveau mondial (Boedeker et al., 2020).

C'est dans ce contexte que nous avons conduit ce travail dans la Préfecture de Meknès au Maroc. La Préfecture de Meknès, est une région caractérisée par des potentialités agricoles très diversifiées qui lui ont permis d'être placée parmi les meilleures zones agricoles du Maroc. Elle enregistre une consommation annuelle dépassant 362 tonnes de produits phytosanitaires (ABH, 2005). L'objectif de cette étude est d'évaluer le mode d'utilisation et de gestion des pesticides par les agriculteurs de cette Préfecture.

\section{Matériels et méthodes \\ Zone d'étude}

Cette étude a été réalisée dans la Préfecture de Meknès, située à 3353'36" Net $5^{\circ} 32^{\prime} 50^{\prime \prime} \mathrm{O}$ (Figure 1). Cette Préfecture compte 835695 habitants. Sa population rurale est de 148120 habitants (RGPH, 2014). La Préfecture se caractérise par un climat méditerranéen semicontinental, dont les hivers sont frais et pluvieux et les étés sont chauds et secs. La surface agricole utile (SAU) au niveau de cette région est de 149516 Ha soit $1,7 \%$ de la SAU totale au niveau national. Le choix de cette aire d'étude est dû à sa forte contribution dans la production agricole du pays et son potentiel de nourrir à la fois ses habitants et ceux de la région de Fès- Meknès.

Les communes étudiées sont Mejjate, Dkhissa, Sidi Sliman Moul Kiffane, Oued Jdida, M’haya, Meknès, Ouislane et Toulal (Figure 1). 


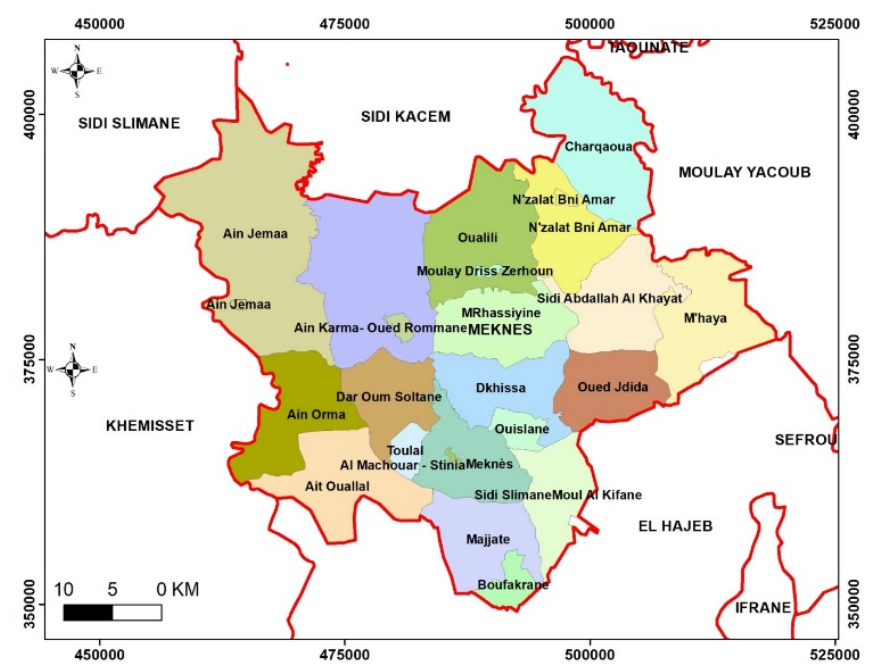

Figure 1. Géolocalisation de la zone d'étude

\section{Unités de recherche et échantillonnage}

Les unités de recherche sont les agriculteurs des zones urbaines, périurbaines et rurales. Au total, 120 agriculteurs à raison de 15 agriculteurs par commune et 40 agriculteurs par zone ont été enquêtés. La technique d'échantillonnage est l'échantillonnage stratifié.

\section{Collecte et analyse des données}

Pour connaître l'utilisation des pesticides dans la Préfecture de Meknès, nous avons soit utilisé une fiche d'enquête pour la collecte des données soit interrogé directement les agriculteurs dans les différentes zones de la Préfecture (zones urbaines, périurbaines et rurales). Les enquêtes ont été réalisées entre le 15 novembre 2020 et le 26 février 2021. Elles consistaient à récolter les données relatives à l'emploi des différents produits chimiques (herbicides, insecticides, fongicides) durant la campagne agricole 2019/2020. Les données collectées ont été saisies et analysées avec le logiciel Microsoft Excel 2010. La statistique descriptive a été utilisée pour calculer les fréquences et le test de Khi2 a été utilisé pour comparer les variables quantitatives. Le seuil de signification retenu était la probabilité $\mathrm{P} \leq 0,05$. Le logiciel ArcGIS 9.3 a été utilisé pour l'élaboration de la carte de la géolocalisation de la zone d'étude (Figure 1).

\section{Résultats et discussions}

\section{Les principales cultures dans la zone d'étude}

L'analyse de la figure 2 fait ressortir que $50 \%$ de la surface agricole lors de l'enquête est consacrée aux céréales, suivies par l'arboriculture (26\%) 
et les légumineuses (13\%). La superficie agricole résiduelle est répartie entre les cultures maraichères, fourragères et les oléagineuses.

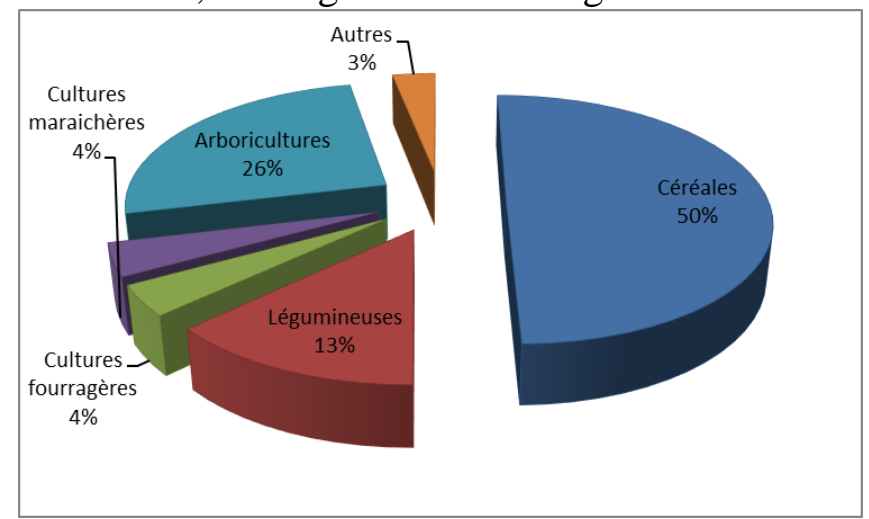

Figure 2. Distribution de la superficie enquêtée par type de culture. Source : enquêtes 20202021

\section{Les principaux pesticides utilisés par les producteurs de la Préfecture de Meknes}

Cette étude révèle que tous les agriculteurs interrogés (100\%) emploient des pesticides. Les pesticides utilisés sont essentiellement des herbicides (44\%) et des fongicides (29\%) (Tableau 1 et Figure 3). Ce résultat corrobore ceux constatés en Amérique (INSERM, 2013) et en Europe (OCDE, 2003). En effet, au Maroc, Maldani et al. (2017) ont rapporté que les herbicides représentaient $57 \%$ de la quantité de pesticides chimiques de synthèse répertoriés. Un résultat similaire a été enregistré à Tadla au Maroc où les herbicides constituaient 33\% des pesticides utilisés (Hafiane et al., 2021). En revanche, ce résultat diverge de celui observé en Algérie où les insecticides et fongicides représentaient $86 \%$ du total des pesticides pulvérisés (Belhadi, 2017).

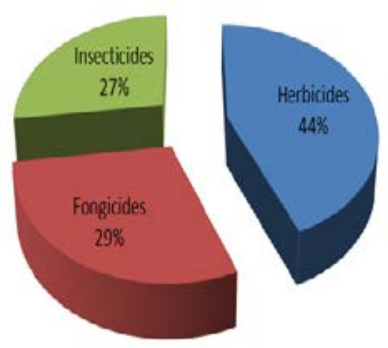

Figure 3. Répartition des pesticides utilisés en fonction des classes.

Source: enquêtes 2020-2021 
Tableau 1. La liste des pesticides les plus couramment utilisés dans la zone d'étude

Source : enquêtes 2020-2021

\begin{tabular}{|c|c|c|c|}
\hline Pesticides & Classe & Famille chimique & Matière active \\
\hline DAKA $50 \mathrm{WP}$ & Herbicides & Urées substituées & Linuron $(50 \%)$ \\
\hline TOYOTO 24 & Herbicides & Diphényl-êthers (dérivés du benzène) & Oxyfluorfene ( $240 \mathrm{~g} / 1)$ \\
\hline FUSILADE FORTE & Herbicides & Azyloxyphenoxypropionique & Fluazifop-p-butyl $(150 \mathrm{~g} / 1)$ \\
\hline GALLANT SUPER & Herbicides & Organophosphorés & Haloxyfop-R-méthyl ester (104 g/1) \\
\hline GRAMOXONE & Herbicides & bipyridinium & Paraquat \\
\hline AGIL & Herbicides & Aryloxyphenoxypropionate & Propaquizafop $(100 \mathrm{~g} / \mathrm{l})$ \\
\hline ALFAHD MIX & Herbicides & Herbicides phénoxy & 2,4-D + MCPA-diméthylamine (DMA) \\
\hline BOOM SUPER & Herbicides & Organophosphorés & Glyphosate -sel d'isopropylamine ( $360 \mathrm{~g} / 1)$ \\
\hline GRANSTAR 75 DF & Herbicides & Sulfonylurées & Tribenuron Methyl (75\%) \\
\hline TORNADO SE & Herbicides & Triazolopyrimidines \& aryloxyacides & 2,4-D (300 g/1) \& Florasulame $(6.25 \mathrm{~g} / 1)$ \\
\hline MEGA 2,4-D & Herbicides & Herbicides phénoxy & 2,4-D -sel d'amine $\quad(840 \mathrm{~g} / 1)$ \\
\hline DAM & Herbicides & Acides phénoxyacétiques & $2,4-\mathrm{D}$-sel d'amine $(400 \mathrm{~g} / 1)$ \\
\hline AGIL & Herbicides & Aryloxyphénoxy propionate & propaquizafop $(10 \%)$ \\
\hline ROUND UP & Herbicides & Organophosphorés & Glyphosate $(450 \mathrm{~g} / 1)$ \\
\hline E1 Afrit 8200 & Herbicides & Aryloxy-acides & 2,4 D-ester butylglycol (200 g/1) \\
\hline CHEVAL ET LION & Herbicides & Aryloxy-acides & 2,4-D-2-éthylhexyl ester $(200 \mathrm{~g} / 1)$ \\
\hline MENJEL 24 EC & Herbicides & Aryloxy-acides & 2,4D d'Ester Butylglcol. \\
\hline AL FAHD MIX & Herbicides & Aryloxy-acides & $2,4-\mathrm{D} 240(\mathrm{~g} / 1)+\mathrm{MCPA} 240(\mathrm{~g} / 1)$ \\
\hline EL GHOUL & Herbicides & Aryloxy-acides & 2,4-D-ester isoctylique $(480 \mathrm{~g} / 1)$ \\
\hline TARZAN 60 & Herbicides & Sulfonylurées & Metsulfuron Methy1 $60 \%$ \\
\hline SEKATOR OD & Herbicides & Sulfonylurées & $\begin{array}{c}\text { Amidosulfuron }(100 \mathrm{~g} / 1)+\text { iodosulfuron }(25 \mathrm{~g} / 1)+ \\
\text { Safner }(250 \mathrm{~g} / 1)\end{array}$ \\
\hline OPUS & Fongicides & Triazoles & Epoxiconazole $(125 \mathrm{~g} / 1)$ \\
\hline PUNCH C & Fongicides & Triazoles, Benzimidazoles & Flusilazole $(250 \mathrm{~g} / \mathrm{l})+$ Carbendazime $(125 \mathrm{~g} / 1)$ \\
\hline APACHE & Fongicides & Triazoles & Propiconazole $(250 \mathrm{~g} / 1)$ \\
\hline BACHLOR $125 \mathrm{SC}$ & Fongicides & Triazoles & Epoxiconazole (125 g/1) \\
\hline SCORE $250 \mathrm{EC}$ & Fongicides & Triazoles & Difenoconazol $(250 \mathrm{~g} / 1)$ \\
\hline FALCON $460 \mathrm{EC}$ & Fongicides & Triazoles & $\begin{array}{c}\text { Tebuconazol }(167 \mathrm{~g} / 1)+\text { Triadimenol }(43 \mathrm{~g} / 1)+ \\
\text { Spiroxamina }(250 \mathrm{~g} / 1)\end{array}$ \\
\hline PROPLANT & Fongicides & Carbamates & Propamocarbe HCL $(722 \mathrm{~g} / 1)$ \\
\hline BAYOUD $10 \mathrm{EC}$ & Fongicides & Triazoles & Penconazole $(100 \mathrm{~g} / 1)$ \\
\hline MATIZ 430 SC & Fongicides & Triazoles & Tebuconazole $(430 \mathrm{~g} / 1)$ \\
\hline CARAMBA $60 \mathrm{SL}$ & Fongicides & Triazoles & Metconazole $(60 \mathrm{~g} / 1)$ \\
\hline TOKRA & Fongicides & Triazoles+ Strobilurines & Penconazole $(87,5 \mathrm{~g} / \mathrm{kg})+$ krésoxim-méthyl $(250 \mathrm{~g} / \mathrm{kg})$ \\
\hline BASAFORT & Fongicides & Carbamates & Zirame $(90 \%)$ \\
\hline ORSALIS & Fongicides & Triazoles & d'Hexaconazole $(50 \mathrm{~g} / 1)$ \\
\hline TAKUMI@ 20WG & Fongicides & Diamides & Flubendiamide $(20 \%)$ \\
\hline TRACTOR $10 \mathrm{E}$ & Insecticides & Pyréthrinoïdes & Alpha-cyperméthrine $(100 \mathrm{~g} / 1)$ \\
\hline MALATHION 50 & Insecticides & Organophosphorés & Malathion $(500 \mathrm{~g} / 1)$ \\
\hline SYNERGY & Insecticides & Organophosphorés \& Pyréthrinoïdes & Chlorpyriphos-éthyl (50\%) + Cyperméthrine (5\%) \\
\hline DECIS PROTECH & Insecticides & Pyréthrinoïdes & Deltamethrine $(15 \mathrm{~g} / 1)$ \\
\hline PIRIMOR 50 DG & Insecticides & Carbamates & Pyrimicarbe $(50 \%)$ \\
\hline DIMETHON & Insecticides & Organophosphorés & Dimethoate $(400 \mathrm{~g} / 1)$ \\
\hline KARATE@ 5 EC & Insecticides & Pyréthrinoïdes & Lambda- Cyhalothrine $(50 \mathrm{~g} / 1)$ \\
\hline ACTARA@ $25 \mathrm{WG}$ & Insecticides & Neonicotinoides & Thiamethoxam $(25 \%)$ \\
\hline PROTEUS & Insecticides & Neonicotinoides+ Pyréthrinoïdes & Thiaclopride $(100 \mathrm{~g} / 1)+(10 \mathrm{~g} / 1)$ Deltamethrine \\
\hline DECIS@ $25 \mathrm{EC}$ & Insecticides & Pyréthrinoïdes & Deltaméthrine \\
\hline DURSBAN 4 & Insecticides & Organophosphorés & chlorpyriphos-êthyl (480 g/1) \\
\hline REEVA 5 EC & Insecticides & Pyréthrinoïdes & Lambda-Cyhalothrine $(50 \mathrm{~g} / 1)$ \\
\hline IMIDAN $50 \mathrm{WG}$ & Insecticides & Organophosphorés & Phosmet $(50 \%)$ \\
\hline
\end{tabular}

D’après la figure 4, les familles chimiques des pesticides les plus identifiés lors de cette enquête sont les triazoles (21\%), les organophosphorés (17\%) et les aryloxyacides (10\%). Ce constat contredit les résultats d'une étude menée au Maroc par le Centre Antipoison et de Pharmacovigilance (CAPM), qui en 2015, a révélé que les organophosphorés représentaient 58\% des pesticides consommés au niveau national (CAPM, 2010). Ce résultat diverge également celui relevé par Maldani et al. (2017) qui ont signalé dans leur enquête que les pesticides de la famille des organophosphorés sont les plus utilisés. 


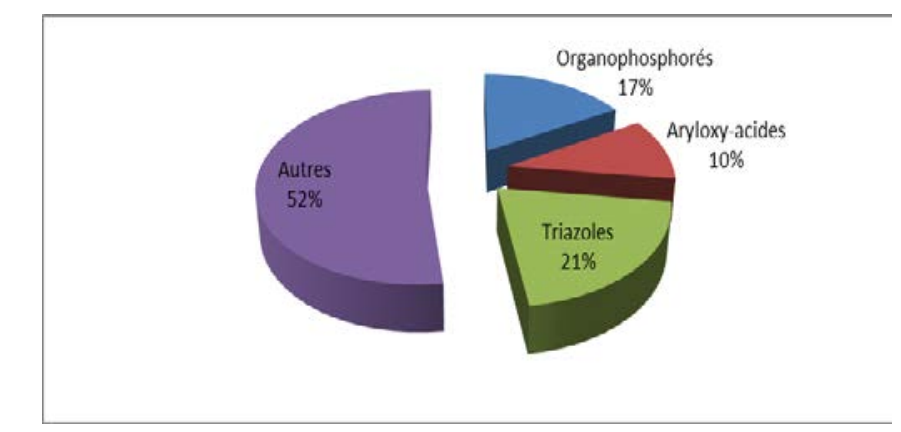

Figure 4. Répartition des pesticides utilisés par les agriculteurs enquêtés en fonction des familles chimiques.

Source: enquêtes 2020-2021

Les résultats d'enquête indiquent que le glyphosate est la matière active la plus utilisée (13\%). En Allemagne, 39\% des terres agricoles exploitées sont traitées avec du glyphosate (Gravert et al., 2013). Au Royaume-Uni, le glyphosate est la matière active la plus consommée (Garthwaite, 2010). En ce qui concerne le Maroc, Maldani et al. (2017) ont constaté que le glyphosate était la matière active la plus répandue (15\%).

\section{Mesures de protection prises par les agriculteurs enquêtés}

L'analyse de la figure 5 montre que plus de la moitié des applicateurs (53\%) de la Préfecture de Meknès n’ont pris aucune disposition de protection. La principale mesure adoptée pendant le traitement est le port du cache-nez (35\%). Les applicateurs qui portent des gants et un cache-nez sont seulement $11 \%$. Enfin, aucun applicateur ne se dote de la tenue de protection complète. Ce résultat est indépendant du niveau d'éducation et de l'ancienneté dans la profession agricole. En effet, le recours au test du Chi-deux pour le croisement des variables niveau d'éducation et non-port de la tenue de protection et entre les variables ancienneté en agriculture et le non-port de la tenue de protection a donné des valeurs $>0,05$. Le faible recours à l'utilisation des équipements de protection par les agriculteurs lors de la pulvérisation des pesticides a été également observé en Côte d'Ivoire par Doumbia et Kwadjo (2009), au Sénégal par Diop (2013), en Algérie par Belhadi (2017), au Burkina Faso par Son et al. (2018) et au Bénin (Ahouangninou et al., 2011 ; 2015 ; Gbonsou et $a l ., 2020)$. Les résultats de l'enquête révèlent également que $22 \%$ des applicateurs consomment de la nourriture ou du tabac durant les opérations de préparation de la bouillie et lors des traitements. 


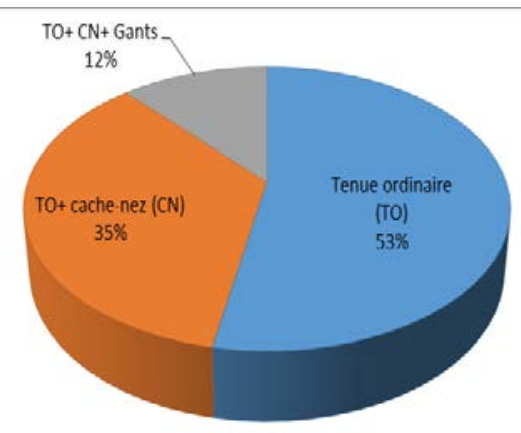

Figure 5. Proportion des moyens de protection utilisés par les agriculteurs enquêtés.

Source : enquêtes 2020-2021

\section{Mesures de protection post-traitement}

Pendant la phase de post-traitement, 59\% des applicateurs se lavaient les mains et se changeaient. Les personnes qui ont fait le bon choix en se douchant ne représentaient que 41\% (Figure 6).

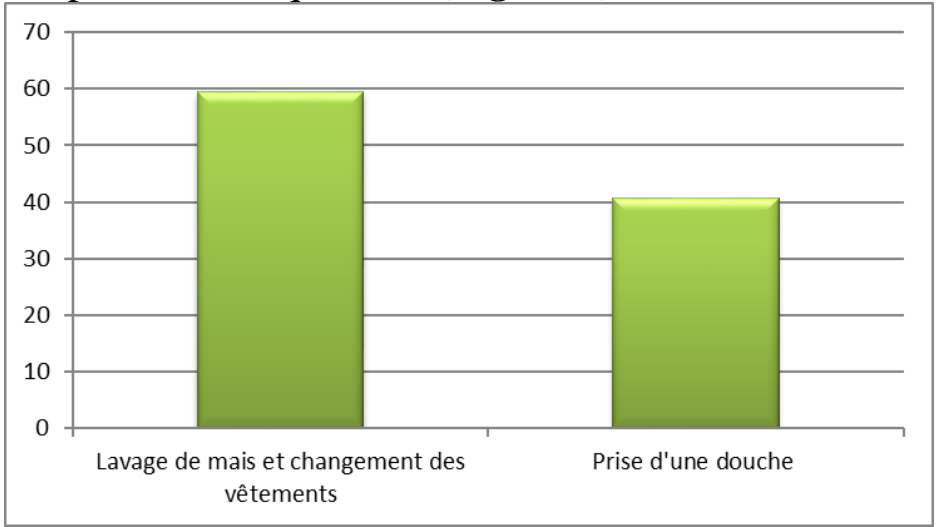

Figure 6. Mesures de protection prises par les agriculteurs enquêtés après la fin des traitements.

Source : enquêtes 2020-2021

\section{Devenir des emballages et contenants usagés}

La figure 7 fournit des informations sur la façon dont les emballages de pesticides sont gérés. Les emballages vides sont incinérés par $45 \%$ et jetés en pleine nature par 42\% des applicateurs. Au Togo, 68\% des emballages vides sont brulés (Kanda et al., 2013), en Côte d'Ivoire, 28\% des maraichers laissent les emballages dans les champs et 52\% les brûlent avec les ordures ou les enfouissent dans le sol (Doumbia et Kwadjo, 2009) et au Benin, 46 \% des producteurs réutilisaient les emballages vides pour se procurer à nouveau des produits phytosanitaires chez les détaillants, $24 \%$ abandonnaient les flacons dans les champs, $10 \%$ les jetaient dans les mares, $2 \%$ les brulaient et $3 \%$ les jetaient à la décharge (Ahouangninou et al., 2011). Au Maroc, une enquête 
réalisée par l'Association d'Education Environnementale et de Protection des Oiseaux au Maroc (SEEPOM), a indiqué que 28\% des agriculteurs des régions de Meknès, Rabat et Tétouan utilisent les emballages vides de pesticides pour le conditionnement des produits alimentaires, entre autres l'eau, 40\% les enfouissent dans le sol, $29 \%$ les incinèrent à l'air libre ou les jettent avec les ordures ménagères et $3 \%$ les abandonnent dans les champs ou les jettent dans la nature (SEEPOM, 2009). A Tadla au Maroc, 53\% des agriculteurs jettent les emballages vides des pesticides, $10 \%$ les incinérèrent et $9 \%$ les réutilisent (Hafian et al., 2021). Dans la région de Doukkala-Abda (Maroc), le recours à l'incinération des emballages est l'usage le plus fréquent chez la majorité des agriculteurs (76\%) (ONSSA, 2015). En Algérie, les emballages vides sont jetés dans la nature par $43 \%$ des agriculteurs et incinérés par 40\% (Belhadi, 2017).

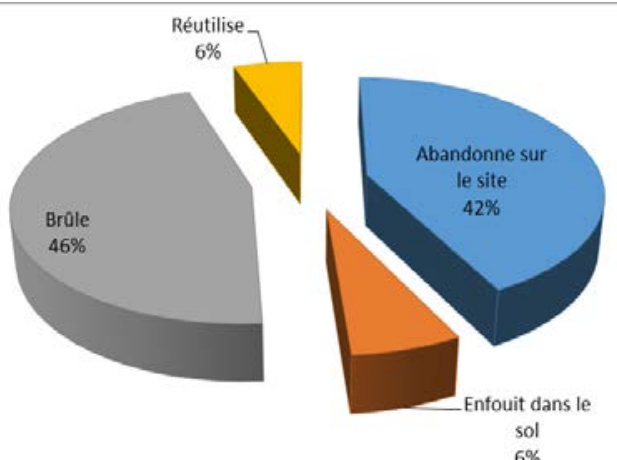

Figure 7. Sort des emballages vides des pesticides utilisés. Source : enquêtes 2020-2021
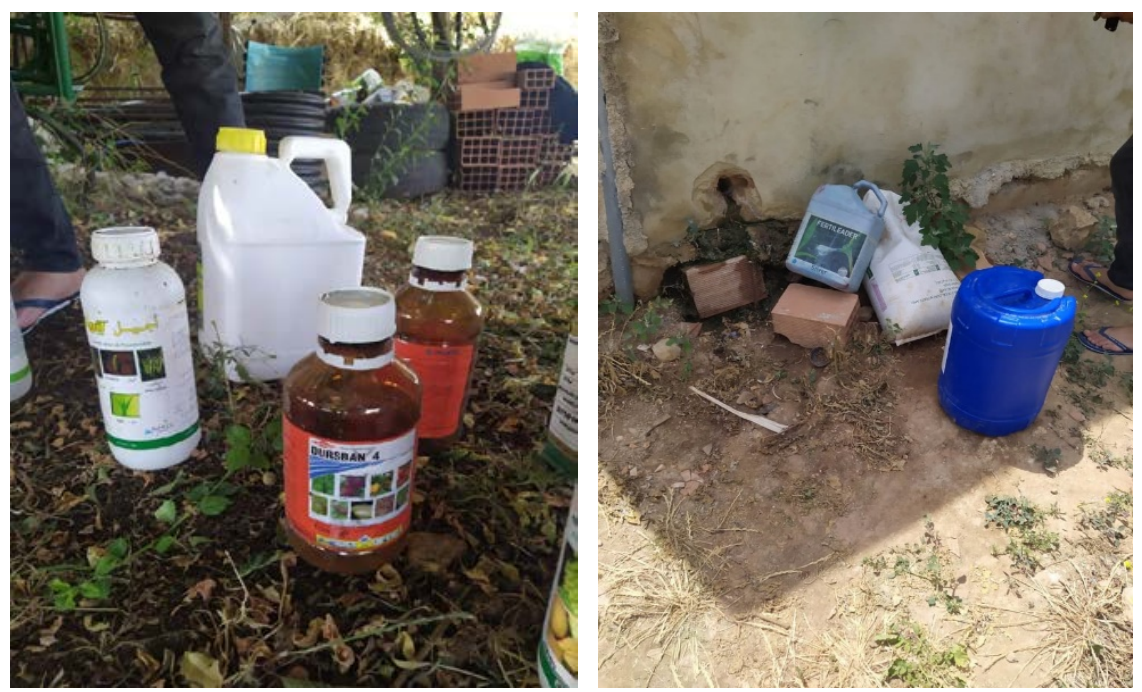

Figure 8. Emballage vide abandonné 


\section{Devenir de la bouillie restante après traitement}

D’après les résultats de cette enquête, deux tiers des agriculteurs interrogés parviennent à utiliser la totalité de la bouillie préparée pour lutter contre les ravageurs des cultures au cours d'une campagne agricole. Près d'un tiers de ces agriculteurs préparent des volumes de bouillie sans les pulvériser entièrement. Cela les amène à déverser l'excédent de bouillie sur le sol. Enfin, une très faible proportion d'agriculteurs ( $3 \%)$ a conservé le reste de la bouillie pour une utilisation ultérieure. Ce résultat est en désaccord avec celui rapporté par Belhadi (2017) qui a constaté que les trois quarts des agriculteurs n'utilisent pas la totalité de la bouillie préparée. Dans la région de DoukkalaAbda au Maroc, 86\% des agriculteurs utilisent toutes les quantités de bouillie préparée et $10 \%$ des agriculteurs éliminent les quantités restantes dans la nature (ONSSA, 2015).

\section{Devenir de l'eau de lavage des pulvérisateurs}

A Meknès, l'eau issue du lavage des pulvérisateurs est déversée sur le sol par 93\% des agriculteurs et seulement 7\% des agriculteurs épandent l'eau du rinçage des pulvérisateurs sur les cultures déjà traitées (Figure 9). En Algérie, l'eau issue du lavage des pulvérisateurs est vidée sur le sol à côté des sources d'eau par 98,5\% des agriculteurs. Seulement 1,5\% des applicateurs répandent l'eau de lavage du pulvérisateur (Belhadi, 2017).

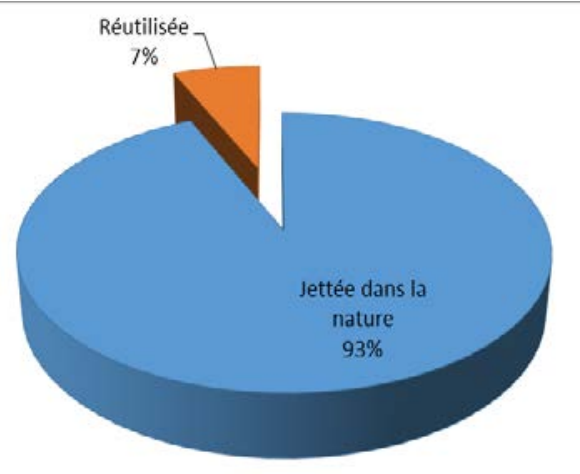

Figure 9. Devenir de l'eau issue du rinçage des pulvérisateurs. Source: enquêtes 2020-2021

\section{Connaissances des instructions d'utilisation des pesticides}

Les résultats de la dernière partie de l'enquête révèlent que $52 \%$ des agriculteurs ignorent les instructions d'utilisation des pesticides (Figure 10). Parmi ces instructions, il faut éviter tout contact des pesticides avec la peau et les yeux, éviter d'inhaler le brouillard de pulvérisation, interdire l'accès à la parcelle traitée à toute personne non impliquée dans les opérations de traitement, aux enfants et au bétail. Lors du traitement, il faut éviter tout 
entraînement du brouillard sur les cultures avoisinantes. Il faut également faire le traitement à un temps calme, ne pas manger, ni boire ou fumer dans les endroits où ces produits sont manipulés, entreposés ou mis en œuvre.

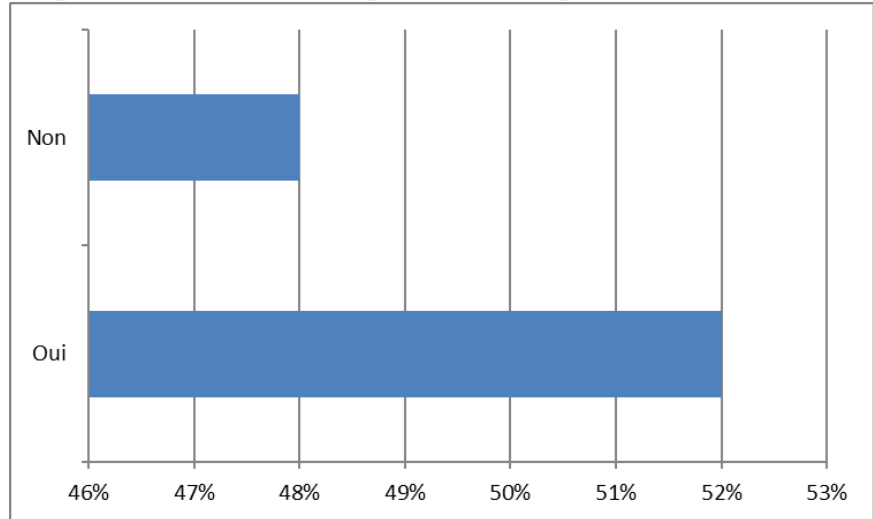

Figure 10. Connaissance des instructions d'utilisation des pesticides.

Source: enquêtes 2020-2021

\section{Respect des doses des pesticides recommandées}

D’après les résultats de l'enquête, $16 \%$ des agriculteurs ne respectent pas les doses des pesticides recommandées (Figure 11). Ce résultat contredit celui rapporté par Naamane et al. (2020) au niveau de la région de CasablancaSettat (Maroc), où $44 \%$ des agriculteurs utilisent les pesticides au hasard. En France, d'après Morillon (2016), 80\% des agriculteurs respectent les doses prescrites et les $20 \%$ restants utilisent des doses inférieures car ils adoptent des techniques alternatives.

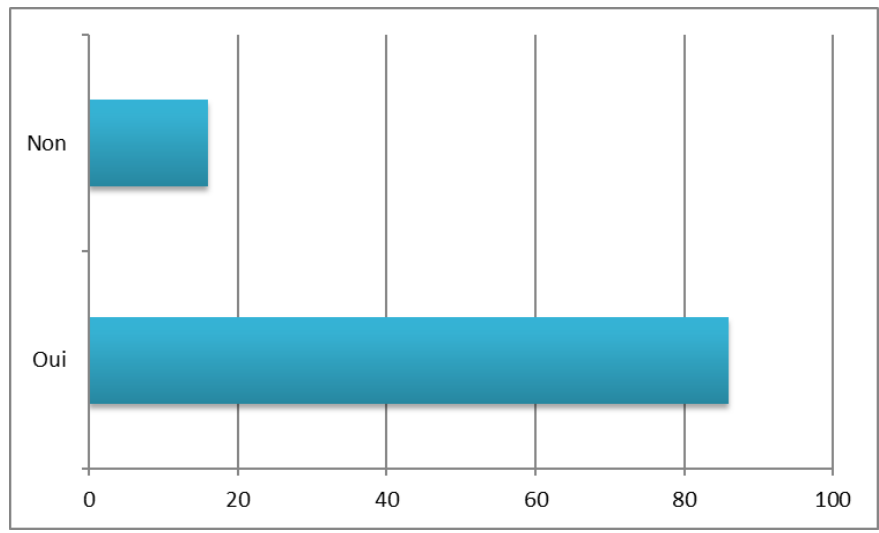

Figure 11. Respect des doses recommandées. Source: enquêtes 2020-2021

\section{Les maladies liées à la manipulation des pesticides citées par les agriculteurs enquêtés.}

Les maladies qui ont été les plus évoquées par les agriculteurs de Meknès suite à l'exposition aux pesticides sont : les troubles de vision, les 
difficultés respiratoires, les irritations cutanées, les maux de tête, les diarrhées, la fatigue, les nausées et les vomissements (Figure 12).

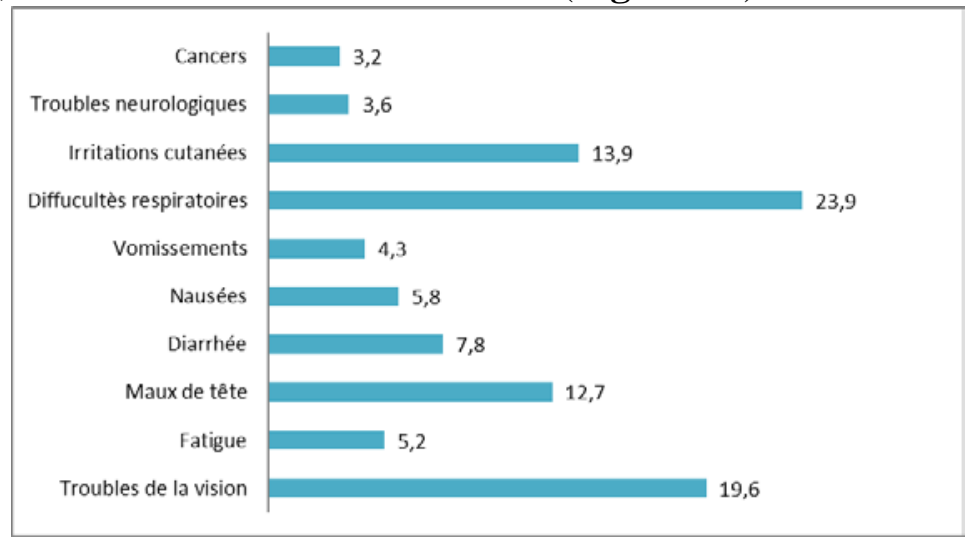

Figure 12. Les maladies les plus évoquées par les agriculteurs de Meknès suite à l'exposition aux pesticides Source: Enquêtes 2020-2021

\section{Conclusion}

La gestion des pesticides avant, pendant et après leur utilisation constitue une problématique environnementale et sanitaire. La production des vivriers (céréales, légumineuses, cultures maraîchères) est soumise à l'utilisation des pesticides à Meknès au Maroc. Les pesticides utilisés sont principalement les herbicides et les fongicides. Ils appartiennent aux familles chimiques des triazoles, des organophosphorés et des aryloxyacides. Le glyphosate est la matière active la plus utilisée. La plupart des applicateurs n'ont pris aucune disposition de protection. Toutefois, le port du cache-nez et des gants est observé chez certains applicateurs pendant le traitement. Pendant les opérations de préparation de la bouillie et de traitements, certains applicateurs consomment de la nourriture ou du tabac. Après le traitement, certains applicateurs ont incinéré les emballages vides alors que d'autres les ont jetés en pleine nature. Environ 59\% des applicateurs se lavaient les mains et se changeaient. La plupart des agriculteurs ignorent les instructions d'utilisation des pesticides. Par conséquent, les applicateurs de Meknès ont souffert des troubles de vision, des difficultés respiratoires, des irritations cutanées, des maux de tête, des diarrhées, de la fatigue, des nausées et des vomissements. Il est crucial de promouvoir la formation des agriculteurs de la Préfecture de Meknès sur l'utilisation judicieuse des produits phytosanitaires par l'organisation des ateliers, des sites de démonstration, porte à porte et la distribution des documentations afin qu'ils adoptent de nouvelles pratiques. Des études complémentaires devraient porter sur le contrôle des résidus de pesticides dans les cultures. Il est indispensable de tester également dans cette zone d'étude, les biopesticides et les techniques de lutte biologique et de 
l'agriculture intégrée, qui ont déjà démontré leur efficacité dans de nombreuses régions du monde et sont moins nocifs pour l'homme et plus écologiques. Le développement de variétés résistantes serait également une piste intéressante pour limiter l'utilisation des produits phytosanitaires.

\section{Remerciement}

Nous remercions l'Académie de Recherche et d'Enseignement Supérieu (ARES) du Gouvernement belge pour le financement de notre séjour posdoctoral à l’Université Catholique de Louvain.

\section{References:}

1. Agence du Bassin Hydraulique de Sebou (ABH). (2005). Mission 1 : "Étude de l'impact de la pollution agricole sur a qualité des eaux de la nappe Fès/Meknès ». Rapport définitif.

2. Assogba-Komlan, F., Anihouvi P., Achigan, E., Sikirou, R., Boko, A., Adje, C., Ahle, V., Vodouhe, R \& Assa A. (2007). Pratiques culturales et teneur en éléments antinutritionnels (nitrates et pesticides) du Solanum macrocarpum au Sud du Bénin. African Journal of Food, Agriculture, Nutrition and Developement $7: 1-21$.

3. Barriuso, E., Benoit, P., Bedos, C \& Charnay, M.P. (2005). Les pesticides dans le sol. Conséquences agronomiques et environnementales. Editions France Agricoles, Paris, 637p.

4. Ahouangninou,C., Fayomi B \& Martin, T. (2011). Évaluation des risques sanitaires et environnementaux des pratiques phytosanitaires des producteurs maraîchers dans la commune rurale de Tori-Bossito (Sud-Bénin). Cahiers Agricultures, 20:216-222.

5. Ahouangninou, C., Martin, T., Cledjo, P., Assogba-Komlan, F., Djogbénou, L., Assogba, B., Soumanou, M.M., Boko, M \& Fayomi, B. (2015). Caractérisation des risques sanitaires et environnementaux des pratiques phytosanitaires dans la production de légumes dans les communes de Cotonou, de Seme-Kpodji et de Ouidah au Sud-Bénin. Cahiers du Centre Béninois de la Recherche Scientifique et Technique, 2: $135-171$.

6. Belhadi A. (2017). Évaluation de la durabilité et étude des pratiques phytosanitaires des exploitations maraîchères sous abri serre d'une région aride : cas des Ziban (Biskra). Thèse de de doctorat en sciences agronomiques, école nationale supérieure agronomique El-Harrach Alger. $324 \mathrm{p}$.

7. Berni, I., Menouni, A., El Ghazi, I., Godderis, L., Duca, RD \& El Jaafari, S. (2021a). Health and ecological risk assessment based on pesticide monitoring in Saïss plain (Morocco) groundwater. 
Environmental Pollution, 276, 116638, ISSN 0269-7491, https://doi.org/10.1016/j.envpol.2021.116638.

8. Berni, I., Menouni, A., El Ghazi, I., Duca, RD., Kestemont, MP., Godderis, L \& El Jaafari, S. (2021b). Understanding farmers' safety behavior regarding pesticide use in Morocco. Sustainable Production and Consumption, $25:$ 471- 483.

9. Boedeker, W., Watts, M., Clausing, P \& Marquez , E. (2020). The global distribution of acute unintentional pesticide poisoning: estimations based on a systematic review. BMC Public Health, 20, 1875. https://doi.org/10.1186/s12889-020-09939-0.

10. Bouzoubaa, A. (2002). Etude de la persistence chimique, la mobilité et l'adsorption du methyl isothiocyanate dans le sol. Thèse de doctorat, Université Mohammed V, Rabat.

11. Brühl, C.A., Pieper, S \& Weber B. (2011). Amphibians at risk? Susceptibility of terrestrial amphibian life stages to pesticides. Environmental Toxicology and chemistry, 30: 2465-2472.

12. Chahine, A. (2011). Modélisation de la dispersion aérienne de pesticides des échelles locales aux échelles régionales, influence des aménagements et quantification des niveaux d'exposition Thèse de Doctorat, Centre International d'Etude Supérieures en Sciences Agronomiques, Montpellier SupAgro, 184p.

13. Carvalho, F.P. (2006). Agriculture, pesticides, food security and food safety. Environmental Science \& Policy, 9 (7-8): 685- 692.

14. Cusaac, J.P.W., Mimbs IV W.H., Belden, J.B., Smith, L.M \& McMurry S.T. (2015). Terrestrial exposure and effects of Headline AMP ${ }^{\circledR}$ Fungicide on amphibians. Ecotoxicology, 24: 1341-1351.

15. Detroux L.(1996). Les produits phytopharmaceutiques : Leurs Objectifs et leurs impacts sur la qualité de la nourriture, de l'eau et de l'environnement. Centre de recherche agronomique, Gembloux.

16. Dinehart, S.K., Smith, L.M., McMurry, S.T., Anderson, T.A.,Smith, P.N \& Haukos, D.A. (2009). Toxicity of a glufosinate and several glyphosate-based herbicides to juvenile amphibians from the Southern High Plains, USA. Science of the Total Environment, 407: 1065-1071.

17. Diop A. (2013). Diagnostic des pratiques d'utilisation et quantification des pesticides dans la zone des Niayes de Dakar (Sénégal). Thèse de doctorat. Université du Littoral Côte d’Opale.190p.

18. Doumbia, M \& Kwadjo, K.E. (2009). Pratiques d'utilisation et de gestion des pesticides par les maraîchers en Côte d'Ivoire : Cas de la ville d'Abidjan et deux de ses banlieues (Dabou et Anyama). Journal of Applied Biosciences, 18 : 992 - 1002.

19. El Azzouzi, E.H., El Bouzaidi, H., Nouri, K., El Azzouzi, M \& Fekhaoui, M. (2014). Study the impact of pesticides using pressure 
indicator and toxicity watershed Merja Zerga (Morocco). Advances in Environmental Biology, 8(17): 31-35.

20. El Bakouri, H. (2006). Développement de nouvelles techniques de détermination des pesticides et contribution à la réduction de leur impact sur les oiseaux par utilisation des substances organiques Naturelles. « Thèse, Université AbdElmalek Saadi, 2006 ».p.9,24, 52.

21. FOCUS (2008). Pesticides in Air: Considerations for Exposure Assessment. Report of the FOCUS Working Group on Pesticides in Air, EC Document Reference SANCO/10553/2006 Rev 2 June 2008. 327 pp.

22. Food and Agriculture Organisation (FAO). (1978). Résidus des pesticides dans les produits alimentaires. Rapport de la réunion conjointe FAO/OMS, Rome.

23. Garthwaite, DG. (2010). Pesticide Usage Survey Report 235: Arable Crops in the United Kingdom,. Food \& Environment Research Agency, DEFRA, UK.

24. Garvert, H., Schmitz, P. M. \& Mirza Nomman, A. (2013), AgroEconomic Analysis of the use of glyphosate in Germany. Outlooks on Pest Management, 24 : 81-85.

25. Gbonsou , A.I.A.,. Adounkpè F. M., Ahouangninou, C., Badou, R. B, Bonou, M.A., Agbossodé J., Adossou E., Atrèvi, N \& Patrick A. Edorh. (2020). Plant protection practices and health impacts in market gardening in South Benin (West Africa), International Journal of Development Research, 10 (10): 40982-40991.

26. Guiral C., Bedos C., Ruelle B., Basset-Mens C., Douzals JP, Cellier P, Barriuso E. (2016) Les émissions de produits phytopharmaceutiques dans l'air. Facteurs d'émission, outils d'estimation des émissions, évaluations environnementales et pesrpectives de recherche Synthèse ADEME 47p.

27. Hafiane, F.Z., El Bouzaidi, H., Nouayti, N,. Tahri, L., El Jarmouni, M., Didi, S and Fekhaoui, M. (2021). Inventory: The pesticides application and its risk assessment in the irrigated perimeter of Tadla-Morocco. Limnological Review, 21 (1) : 15-27.

28. Houze, E. (2003). Mesure des pesticides dans l'air ambiant en milieu urbain, rapport Air Breizh, 78 p.

29. Idrissi, M., Aït Daoud, N., Ouammi, L., Rhalem, N., Soulaymani, A and Soulaymani Bencheikh, R. (2010). Toxicologie Maroc, $n^{\circ} 4,1$ trimmestre : 5-7.

30. Institut national de la santé et de la recherche médical (INSERM). (2013). Pesticides: Effets sur la sante, synthèses et recommandations. Rapport. Paris : XII-1001 p. 
31. Kanda, M., Djaneye-Boundjou, G., Wala, K., Gnandi, K., Batawila, K., Sanni, A \& Akpagana, K.. (2013). Application des pesticides en agriculture maraichère au Togo. VertigO - la revue électronique en sciences de l'environnement [En ligne], Volume 13 Numéro 1 | avril 2013, mis en ligne le 11 avril 2013. (Consulté le 27 juillet 2021), [en ligne], adresse URL: http://vertigo.revues.org/13456 ; DOI : 10.4000/vertigo.13456.

32. Maldani, M., Dekaki, EM., Nassiri, L and Ibijbijen, J. (2017). State of Art on the Use of Pesticides in Meknes Region, Morocco. American Journal of Agricultural Science, 4 (6) :138-148.

33. MAPM - L'agriculture marocaine en chiffres, 2014. Ministère de l'alimentation, de l'agriculture et de la pêche (MAAP). (2009). Les politiques agricoles à travers le monde - Quelques exemples Collection 2015 - Maroc.

https://www.agropolis.fr/formation/pdf/2009-politique-agricolemonde-maroc.pdf. Consulté le 23 Août 2021.

34. Mojtahid A. (2006). Nouveau contexte phytosanitaires, adaptation du secteur. Agricultue du Maghreb. 14 : 63-66.

35. Morillon A. (2016). Les risques liés à l'utilisation des pesticides: Enquête auprès des agriculteurs du Poitou Charentes. Thèse de Doctorat en pharmacie. Université de POITIERS, Faculté de Médecine et de Pharmacie.108 p.

36. Naamane, A., Sadiq, A., Belhouari, A., Iounes N \& El Amran, S. (2020). Enquête sur l'utilisation des engrais et pesticides chez les agriculteurs de la région de Casablanca-Settat. Revue Marocaine des Sciences Agronomiques et Vétérinaires, 8(3): 279-285.

37. Office National de Sécurité Sanitaire des produits Alimentaires (ONSSA). (2015). etude sur le suivi de l'effet des pesticides sur la santé humaine et l'environnement, $73 \mathrm{p}$.

38. Pazou, EYA., Laleye, P., Boko, M., Van Gestel, CAM., Ahissou, H., Akpona, S., van Hattum, B., Swart, K \& van Straalen, NM. (2006). Contamination of fish by organochlorine pesticide residues in the Ouémé river catchment in the Republic of Bénin. Environment International, 32 : 594-9.

39. Raffa, C.M \& Chiampo, F. (202). Bioremediation of Agricultural Soils Polluted with Pesticides: A Review. Bioengineering, 8 (92) :1-29.

40. Saadane, O. (2018). Impact des pesticides sur l'environnement et la santé humaine et méthodes alternatives. Thèse de doctorat en médecine, Université Mohammed V, Rabat, 163p.

41. SEEPOM. (2009, juin). L'utilisation des emballages vides des pesticides pour le stockage des aliments et de l'eau nuit à la santé 
[affiche]. Séminaire National sur la Gestion des Emballages Vides de Pesticides au Maroc, Rabat, Maroc

42. Son, D., Zerbo, K.B.F., Bonzi, S., Legreve, A., Somda, I., Schiffers, B \& Legreve, A. (2018). Assessment of Tomato (Solanumlycopersicum L.) Producers' Exposure Level to Pesticides, in Kouka and Toussiana (Burkina Faso). International Journal of Environmental Research and Public Health, 15, 204.

43. Van Jaarsveld, J.A \& Van Pul. (1999). Modelling of Atmospheric Transport and Deposition of Pesticides. Water, Air Soil Pollut., 115 : $167-182$.

44. Wade CS. (2003). L'utilisation des pesticides dans l'agriculture périurbaine et son impact surl'environnement. Thèse de Pharmacie, Dakar.http://indexmedicus.afro.who.int/iah/fulltext/wade_cheikh.pdf. WHO, UNEP, editors. Public health impact of pesticides used in agriculture. Geneva: World Health Organization; 1990. p. 128. Available from:

https://apps.who.int/iris/bitstream/handle/10665/39772/9241561394.p df?sequence=1\&isAllowed=y. [cited 2020 Mar 12]. 